\title{
An Update on Microinvasive Glaucoma Surgery Concurrent With Cataract Extraction
}

\author{
Sarah H. Van Tassel ${ }^{1} \cdot$ Darren Chen ${ }^{2}$ \\ Published online: 25 November 2019 \\ (C) The Author(s) 2019
}

\begin{abstract}
Purpose of Review In this article, we review the current options for microinvasive glaucoma surgery (MIGS) combined with cataract extraction in the USA and the efficacy of the various approaches.

Recent Findings MIGS is a rapidly growing area of research and clinical interest. Available surgeries aim to reduce intraocular pressure by improving aqueous outflow through the trabecular or subconjunctival pathways or by reducing aqueous production. Summary Multiple approaches to MIGS are utilized in clinical practice. On average, these surgeries are safe and modestly effective in reducing intraocular pressure. Future research will demonstrate which surgical approaches are most efficacious, whether and how to combine MIGS, and the role of MIGS in improving patient quality of life.
\end{abstract}

Keywords Glaucoma $\cdot$ Cataract $\cdot$ Microinvasive glaucoma surgery $\cdot$ Glaucoma treatment $\cdot$ Intraocular pressure

\section{Introduction}

Glaucoma is a progressive polyfactorial optic neuropathy that causes ganglion cell apoptosis and visual field loss. Glaucoma affects more than 70 million people worldwide $[1,2]$ and can progress to blindness.

Reduction of intraocular pressure (IOP) has been shown to slow glaucomatous progression, and IOP is the primary treatment target [3]. Both glaucoma and its treatment can significantly effect quality of life, even in early glaucoma. [4-7] This has necessitated the search for IOP lowering interventions that are effective, low risk, and can be employed in early disease.

\section{Microinvasive Glaucoma Surgery}

The historical mainstays of glaucoma treatment are topical or oral IOP lowering therapy, laser trabeculoplasty, and

This article is part of the Topical Collection on Cataract

Sarah H. Van Tassel

sjh2006@med.cornell.edu

1 Department of Ophthalmology, Weill Cornell Medicine, 1305 York Avenue, 11th floor, New York, NY 10021, USA

2 Weill Cornell Medical College, New York, USA incisional glaucoma surgery (trabeculectomy and glaucoma drainage device implantation). Over the last 15 years, microinvasive glaucoma surgery (MIGS) has emerged as a new family of glaucoma treatment options (Table 1). Five key features define MIGS: (1) ab interno approach; (2) minimal trauma to the target tissue; (3) modest efficacy; (4) excellent safety; and (5) rapid recovery with minimal impact on quality of life [8••].

The importance and promise of MIGS is perhaps best demonstrated by the preferences of glaucoma surgeons themselves. An anonymous survey was distributed to members of the American Glaucoma Society (AGS), and participants were asked to adopt the role of a patient with primary open angle glaucoma (POAG) and progressive visual field loss in need of glaucoma surgery [9•]. Overall, ab interno trabeculotomy (gonioscopy-assisted transluminal trabeculotomy [GATT] or Trab360 [Sight Sciences, Menlo Park, CA]) was the most frequently chosen procedure $(20.3 \%)$. MIGS in aggregate was selected in over half of responses (53.2\%) [9•]. Most interestingly, only $17.6 \%$ and $6.9 \%$ of respondents preferred to have a trabeculectomy or glaucoma drainage implant, respectively, as a primary procedure despite the 2016 AGS member survey of surgical practice preferences demonstrating that approximately $59 \%$ and $23 \%$ of AGS members chose to offer a trabeculectomy or glaucoma drainage implant, respectively, to a hypothetical patient with POAG and no prior surgery $[9 \bullet]$. 
Table 1 MIGS currently available in the USA

\begin{tabular}{l} 
Mechanism of IOP reduction: \\
\hline Aqueous production \\
Endocyclophotocoagulation \\
Aqueous outflow \\
Trabecular \\
iStent \\
iStent inject \\
Hydrus \\
Kahook dual blade \\
Trabectome \\
Gonioscopy-assisted transluminal trabeculotomy \\
Ab interno canaloplasty \\
OMNI system (viscodilation and trabecular unroofing) \\
Subconjunctival \\
Xen gel stent \\
Suprachoroidal \\
No device available
\end{tabular}

MIGS is one of the fastest evolving topics in all of ophthalmology. Herein, we wish to highlight some of the newest additions to the peer-reviewed literature published in 20182019 that contributed to the field and to our care of patients; we also include our opinions about best practices. We will emphasize papers examining MIGS together with cataract extraction. systematic reviews of the MIGS landscape have been published elsewhere [10•].

\section{Current Options}

\section{iStent and iStent Inject}

The iStent (Glaukos Corporation, San Clemente, CA) was approved by the FDA in 2012 and was the first ab interno glaucoma implant to be approved in the USA. The iStent inject (Glaukos Corporation) second generation device was approved by the FDA in June 2018 [11]. Both devices are approved for use in patients with mild to moderate POAG and visually significant cataract and have good safety profiles.

Wang et al. investigated whether implantation of the iStent concurrent with cataract surgery was associated with a reduction in the use of ocular antihypertensive medications in a US health care claims database [12]. Patients undergoing bilateral iStent and cataract extraction had a greater mean reduction in drops used (0.99 vs. 0.49 ; postoperative month 20-24; $P<0.001)$ and a higher proportion receiving no drops postoperatively $(73.5 \%$ vs. $55.3 \%$, postoperative month $20-24$; $P<0.001)$ compared to matched controls undergoing cataract extraction alone [12], providing real-world evidence that
iStent placement concurrent with cataract extraction moderately reduces prescriptions of glaucoma medication.

The two year results of the pivotal iStent inject trial were released this year, demonstrating that $75.8 \%$ of iStent inject with cataract extraction eyes vs. $61.9 \%$ of cataract surgery alone eyes experienced $\geq 20 \%$ reduction from baseline in unmedicated diurnal IOP $(P=0.005)$ [13]. The mean reduction in washout diurnal IOP was greater in iStent inject eyes $(7.0 \pm$ $4.0 \mathrm{mmHg})$ than in control eyes $(5.4 \pm 3.7 \mathrm{mmHg} ; P<0.001)$. Finally, more iStent inject eyes achieved an unmedicated diurnal IOP $\leq 18 \mathrm{mmHg}$ (63.2\% vs. $50.0 \%$ of control eyes) [13].

In a novel line of inquiry, Gillmann and colleagues performed anterior segment optical coherence tomography (ASOCT) on 25 eyes of 19 subjects following implantation of 2 iStent inject devices combined with cataract surgery to assess device position [14]. Ninety-two percent of devices were visible on AS-OCT compared with $88 \%$ of devices visible by gonioscopy. Among gonioscopically visualized devices, ASOCT showed that $54.3 \%$ had a protruding portion into the anterior chamber; the openings of the remaining devices were positioned under the trabecular surface despite being visible by gonioscopy. Not surprisingly, burying of the iStent inject device was associated with higher postoperative IOP [14].

A recent Cochrane Review investigated whether implantation of one or more iStent or iStent inject devices ("iStents") reduced IOP or eye drop burden or changed patient quality of life compared with conventional medical, laser, or surgical treatments [15]. The Glaukos Corporation provided funding and sponsorship for all the randomized controlled trials that met criteria for inclusion in their review [15]. The authors concluded that there is low-quality evidence that iStent implantation may result in higher proportions of participants who are drop-free or achieve lower IOP [15]. None of the reviewed studies provided information on patient quality of life. The lack of high-quality evidence led authors to conclude that clinical decisions about iStent use should be based on surgeon judgment and patient preference [15].

Finally, Huang and colleague demonstrated that placement of iStent injects in regions of high or low aqueous humor outflow resulted in different patterns of angiographic aqueous humor outflow [16]. An improved understanding of how to recruit low outflow regions or enhance high outflow regions may allow surgeons to improve the success of MIGS surgery in the future.

\section{Hydrus Microstent}

The Hydrus microstent (Ivantis, Inc., Irvine, CA) facilitates aqueous outflow through the conventional pathway. The nitinol stent is threaded into and dilates about 90 degrees of Schlemm canal and includes a lumen for trabecular meshwork bypass. FDA approval in 2018 was based on 24 month results of the prospective, multicenter, randomized controlled 
HORIZON trial, the largest MIGS trial to date [17••]. Subjects with concomitant POAG and cataract were randomized to receive a Hydrus microstent or no stent following uncomplicated cataract extraction. Results from 556 eyes showed that unmedicated modified diurnal IOP was reduced by $\geq 20 \%$ in $77.3 \%$ of eyes in the Hydrus group compared to $57.8 \%$ of eyes in the no stent group (a primary endpoint, $P<0.001$ ) and $\geq$ $30 \%$ in $53.4 \%$ of Hydrus patients vs. $32.1 \%$ of phacoemulsification patients $(P<0.0001)[17 \bullet \cdot]$. On average, the device reduced unmedicated modified diurnal IOP by about $7.6 \mathrm{mmHg}$, approximately $2.3 \mathrm{mmHg}$ more that the phacoemulsification alone group [17••].

Consistent with results of the pivotal trial, a recent review examining complications in MIGS revealed that complications with Hydrus microstent implant are infrequent and include peripheral anterior synechiae formation, hyphema, and elevated IOP [18]. Rare stent malpositioning was also reported [18].

In a small pilot study in which IOP was monitored using a contact lens sensor in patients with open angle glaucoma, Hydrus implantation was associated with smaller signal fluctuations compared with medical treatment [19], a potentially promising observation given the available evidence suggesting that larger diurnal IOP fluctuations may be a risk factor for glaucoma progression [20]. Cataract surgery was not concurrent with Hydrus implantation in this study [19].

\section{Kahook Dual Blade}

The Kahook Dual Blade (KDB, New World Medical Inc., Rancho Cucamonga, CA) is a disposable goniotomy device designed to excise trabecular meshwork. The sharp, tapered tip allows the blade to enter through the trabecular meshwork into Schlemm canal. The ramp then elevates the trabecular tissue, and the dual blades make parallel incisions thereby removing a section of trabecular meshwork and exposing the associated collector channels [21].

There is a growing body of evidence demonstrating the efficacy of goniotomy with the KDB and highlighting its potential role in treating eyes with severe glaucoma and angle closure glaucoma; such eyes are generally excluded from the above-mentioned trabecular device trials. In a retrospective series of combined phacoemulsification and KDB in 42 eyes from 36 patients with severe-stage glaucoma, $64.3 \%$ of eyes had achieved IOP $\leq 15 \mathrm{mmHg}$ without additional glaucoma procedures [22]. The mean IOP reduction was $2.1 \pm$ $4.67 \mathrm{mmHg}(P=0.022)$, and mean medication reduction was $1.2 \pm 1.4(P \leq 0.001)$ [22].

In a study of 42 eyes of 24 subjects with angle closure glaucoma undergoing phacoemulsification and KDBassisted goniosynechialysis and excisional goniotomy, 6 months of follow-up demonstrated mean IOP reductions of $10.1-12.8 \mathrm{mmHg}(38.4-49.5 \% ; P<0.0001)$ [23]. At
6 months, $92.9 \%$ of eyes achieved IOP $\leq 18 \mathrm{mmHg}$, all eyes achieved IOP reduction of $\geq 20 \%$, and $85.7 \%$ were medication-free [24]. Although there was no comparison to cataract surgery alone, their findings suggest that goniosynechialysis and excisional goniotomy with the KDB together with cataract extraction may play a helpful role in optimizing outflow and IOP reduction in angle closure glaucoma eyes.

\section{Trabectome}

Trabectome (NeoMedix Corporation, Tustin, CA) was introduced in 2004 as the first MIGS device. The Trabectome handpiece needle tip pierces the trabecular meshwork and then circumferentially ablates the trabecular meshwork for 3 to 6 clock hours. It is approved in the USA for use with and without concurrent cataract surgery and across the spectrum of disease severity and subtypes [25].

Using success criteria of IOP $\leq 21 \mathrm{mmHg},>20 \%$ reduction from preoperative IOP, and no need for further glaucoma surgery, a 5 year study of combined phacoemulsification and Trabectome surgery demonstrated success of 67.5\% [26]. Risk factors for failure were lower baseline IOP, younger age, and higher central corneal thickness. Exfoliative glaucoma was associated with a higher success rate [26]. In a series of eyes undergoing Trabectome with or without cataract extraction, IOP lowering was similar in eyes with mild compared to moderate to severe glaucoma [25].

There is growing interest in whether race/ethnicity alters MIGS efficacy. In a study comparing the IOP lowering effect of Trabectome with or without cataract extraction in 82 African American and 82 Caucasian eyes, there was a significant reduction in IOP in both groups and no difference between the 2 groups in IOP, number of medications, and complications [27].

\section{Gonioscopy-Assisted Transluminal Trabeculotomy}

Gonioscopy-assisted transluminal trabeculotomy (GATT) was first described in 2014 [28] and is most commonly performed using a $5-0$ prolene suture (Ethicon, Inc., Somerville, NJ) or illuminated microcatheter (Ellex, Adelaide, Australia) to cannulate Schlemm canal. Once the distal tip of the suture or catheter has traversed the entire circumference of the canal, it is externalized, creating a 360 degree trabeculotomy.

Grover et al. reported the 2 year results of 198 patients with various open angle glaucoma subtypes who underwent GATT with and without cataract extraction [29]. Eyes with secondary open angle glaucoma had a greater mean percentage of IOP decrease $(49.8 \%)$ than eyes with POAG $(37.3 \%)$. Additionally, eyes with POAG that were already pseudophakic at the time of GATT tended to have a higher 
cumulative proportion of failure and a higher cumulative proportion for reoperation after the 2 year time point [29].

The extent of the episcleral venous fluid wave observed at the completion of GATT surgery may be a prognostic indicator of surgical success [30], emphasizing the importance of the distal outflow system in MIGS.

\section{Ab Interno Canaloplasty and Viscodilation}

Canaloplasty with a tensioning suture and dilation of Schlemm canal with viscoelastic devices were both pioneered as ab externo approaches and remain in use [31]. Ab interno approaches to these technique are a growing area of interest, and $\mathrm{ab}$ interno canaloplasty $(\mathrm{ABiC})$ using the iTrack microcatheter was demonstrated in a series of 75 eyes to significantly reduce both intraocular pressure $(20.4 \pm 4.7 \mathrm{mmHg}$ to $13.3 \pm 1.9 \mathrm{mmHg}$ ) and ocular antihypertensive medication use $(2.8 \pm 0.9$ medications to $1.1 \pm 1.1$ medications, both $P<0.0001)$ as a standalone and in combination with cataract extraction [32]. In contrast to GATT, in which the iTrack is externalized to create a trabeculotomy, $\mathrm{ABiC}$ involves circumferential intubation of Schlemm canal and then subsequent slow withdrawal of the catheter while steadily injecting viscoelastic device through the catheter for dilation of the Schlemm canal and the distal outflow pathways.

The OMNI Glaucoma Treatment System (Sight Sciences, Menlo Park, CA) entered the market in 2018 as a combination of the company's previous Trab360 and Visco360 devices, and a prospective trial in combination with cataract extraction is currently underway [33]. Similar to ABiC, the OMNI can be used to viscodilate Schlemm canal; it can also unroof the trabecular meshwork.

\section{XEN Gel Stent}

The XEN gel stent (Allergan plc, Dublin, Ireland) is an ab interno gelatin stent with a $45 \mu \mathrm{m}$ lumen that is implanted into the subconjunctival space to bypass the trabecular outflow pathway and connect aqueous with a subconjunctival filter. It is the only currently available subconjunctival MIGS device and is indicated in primary open angle glaucoma and pseudoexfoliative or pigmentary glaucoma with open angles. Multiple approaches to antimetabolite use with the XEN gel stent have been described [34], as have multiple techniques for implantation, including off-label ab externo approaches with and without conjunctival incisions. Needling revision, often with antimetabolite, is sometimes necessary following XEN gel stent placement to reduce the risk of bleb failure due to fibroblast proliferation and episcleral fibrosis.

Multiple recent series have demonstrated the safety and efficacy of XEN gel stent placement at the time of cataract surgery in reducing IOP and IOP lowering medication [35-42]. Interestingly, Widder et al. reported that pseudophakic eyes had the most favorable primary success rate compared to phakic eyes and combined surgery with phacoemulsification, and this finding influenced their clinical decision making so as to no longer offer patients combined surgery [43].

Of note, additional lumen sizes of the XEN gel stent have been investigated, such as the $63 \mu \mathrm{m}$ stent [44] but are not available for implantation in the USA.

\section{Endocyclophotocoagulation}

In contrast to some of the newer outflow-targeted procedures described above, endocyclophotocoagulation (ECP; Endo Optiks, Little Silver, NJ) has been available for decades [45] and targets the ciliary processes to decrease aqueous humor production and thus lower intraocular pressure. This tissue destruction inherent in ECP has led some surgeons to exclude ECP from the MIGS category. Overall, the safety and efficacy of ECP is well established.

Prolonged postoperative inflammation is a feared side effect of ECP. Given the increasing trend toward use of ECP in eyes with good vision and mild to moderate glaucoma, Edmiston et al. sought to compare the incidence of persistent anterior uveitis after combined phacoemulsification and ECP by race and to describe its impact on visual acuity or IOP [46•]. Persistent anterior uveitis was defined as the presence of anterior chamber cells or a continued need for steroids at the three month postoperative visit. Persistent anterior uveitis was present in $22.4 \%$ of patients and was both more common and more symptomatic in African Americans compared with whites. While persistent inflammation was common, there was no significant difference in visual acuity or IOP reduction in those without inflammation, those with untreated inflammation, and those with inflammation treated with topical steroids. Interestingly, among those patients with persistent inflammation at month three, $44 \%$ had worsened $(18 \%)$ or no improvement (26\%) in inflammation at week 1 compared with day 1 postoperatively, suggesting a role for early and aggressive treatment of these patients. The investigators concluded that surgeons should employ caution when offering ECP to African American patients but that inflammation was not a poor prognostic sign after ECP. Although their study was not intended to evaluate ECP efficacy, their patients had a modest IOP reduction of about $2 \mathrm{mmHg}$ and required about one half drop less than preoperatively [46•].

In a retrospective chart review of 63 eyes that underwent phacoemulsification and ECP, Lin et al. sought to investigate the differential efficacy in eyes with POAG vs. chronic angle closure glaucoma (CACG) [47]. The 22 eyes with CACG had greater IOP reduction and medication reduction than the 41 eyes with POAG at 1 year (6.4 vs. $2.1 \mathrm{mmHg}, P=0.01 ; 0.9$ vs. 0.2 medications, $P=0.04)$ and at final follow-up (6.2 vs. $2.4 \mathrm{mmHg}, P=0.02 ; 0.9$ vs. 0.3 medications, $P=0.05$ ). IOP 
reduction and medication reduction were no different for eyes with mild, moderate, or advanced glaucoma at either time point [47]. This finding may be partially accounted for by the additional benefit of cataract extraction in eyes with CACG but leaves open the possibility that ECP may have an added benefit in CACG eyes.

Two recent Cochrane reviews explored the role of ECP. Toth and colleagues found insufficient high-quality evidence on the effects of ECP for OAG and primary angle closure [48]. Reviewing both ECP and transscleral cyclophotocoagulation, Chen et al. were not able to find conclusive evidence to support whether cyclodestructive procedures for refractory glaucoma result in better outcomes and fewer complications than other glaucoma treatments or whether one type of cyclodestructive procedure is superior [49].

\section{Comparative Studies}

Two recent studies compared the efficacy of goniotomy with the KDB to iStent implantation in glaucoma patients undergoing cataract surgery. In a retrospective multicenter comparison of 237 eyes undergoing KDB goniotomy with phacoemulsification and 198 eyes undergoing iStent implantation with phacoemulsification, authors reported that those in the KDB group had a significantly greater IOP reduction ($4.2 \mathrm{mmHg}, 23.7 \%$ ) compared to the iStent implantation and phacoemulsification group $(-2.7 \mathrm{mmHg}, 16.4 \%, P<0.001)$ after six months of follow-up in eyes with mild to moderate glaucoma [24].

Another retrospective study of 77 eyes with mild POAG demonstrated a slightly higher average IOP reduction of $14.3 \%$ in the iStent group $(n=48)$ compared with $12.6 \%$ in the KDB group $(n=29)$, although this did not reach statistical significance [50]. Both groups experienced a statistically significant reduction in IOP and a reduction in IOP lowering medications with excellent safety at 12 months [50].

In April 2019, Ivantis announced the results of the first prospective, multicenter, randomized clinical trial comparing the efficacy of two MIGS devices: the Hydrus microstent and two iStent inject stents [51••]. Included eyes had POAG, pseudoexfoliation glaucoma, or pigmentary glaucoma. Device implantation was not combined with cataract extraction. At 12 months, $47 \%$ of eyes in the Hydrus group were medication-free compared to $24 \%$ of eyes in the iStent inject group; medication washout was not performed. Among eyes off IOP lowering therapy, mean IOP without medications was $17.3 \pm 3.3 \mathrm{mmHg}$ in the Hydrus group and $19.2 \pm 2.4 \mathrm{mmHg}$ in the iStent group $[51 \bullet \bullet$.

In a single surgeon retrospective series comparing iStent and Hydrus in combination with cataract extraction, both devices lowered IOP and medication use more than phacoemulsification alone [52]. The Hydrus patients were on significantly fewer medications $(0.5 \pm 0.2$ medications $)$ at 12 months than the iStent patients $(P=0.03)$ [52].

In a retrospective matched comparison of Trabectome or iStent placement in combination with cataract surgery, at 24 months investigators observed that $53 \%$ of Trabectome eyes and $16.6 \%$ of iStent eyes achieved IOP of $21 \mathrm{mmHg}$ or less without medications $(P<0.05) ; 17.6 \%$ of Trabectome eyes and no iStent eyes had an IOP reduction of $20 \%$ or more without medication [53].

\section{Combination MIGS}

Although of interest in clinical practice, scant studies have accessed the role of combining MIGS [54], and only limited evidence is available to demonstrate whether combining MIGS is additive with respect to IOP control [55]. In our experience, ECP can be combined with outflow-targeted procedures such as KDB goniotomy to optimize IOP reduction, and this can be done cautiously but successfully in eyes with severe disease for whom the alternative might be incisional surgery. We have found this to be particularly helpful in eyes with shorter axial lengths and CACG. A potential drawback of combination MIGS is limitations with respect to insurer reimbursements, and this must be handled on an individualized basis in conversation with the payor, patient, and surgery center.

\section{Pipeline Options}

New MIGS devices are in the pipeline, such as the iStent Supra (Glaukos), which is in US clinical trials [56]. Additional devices and new surgical techniques will enhance cataract surgeons' armamentarium for reducing IOP at the time of cataract extraction.

\section{Preoperative Management}

Chronic topical IOP lowering medications are a significant risk factor for trabeculectomy failure due to chronic inflammatory changes in the conjunctiva that increase fibrosis and scarring [57, 58]. Owing to this increased risk of failure, some surgeons recommend topical antiinflammatory treatments with or without a topical glaucoma medication holiday prior to trabeculectomy; a survey of UK glaucoma specialists revealed that this practice is viewed as "necessary" by about half of respondents and "beneficial" by $85.9 \%$ [59]. Many surgeons are applying these same pretreatment principles to patients who will be undergoing placement of a Xen gen stent. Depending on the degree of conjunctival and 
eyelid margin inflammation, patients can be started on topical steroids three or more days preoperatively and may also benefit from cessation of topical IOP lowering therapy (replaced with an oral carbonic anhydrase inhibitor, when appropriate), transition to preservative free topical medications, oral doxycycline, eyelid hygiene in the form of lid scrubs and warm compresses, and other ocular surface treatments.

Just as selective laser trabeculoplasty has been hypothesized to be more effective in treatment-naïve eyes [60], we hypothesize that chronic topical glaucoma therapy and ocular surface disease may also affect the success of MIGS surgeries targeting the traditional outflow pathway. These surgeries reduce or bypass outflow resistance at the trabecular meshwork, where the majority of outflow resistance occurs. Downstream, the episcleral and conjunctival vessels are responsible for distal outflow. Subconjunctival inflammatory changes may increase resistance in the episcleral pathway and impede surgical success despite a reduction in trabecular resistance. Whether a brief period of preoperative steroid or escalated ocular surface disease treatment has any effect on MIGS success has not been demonstrated.

\section{Postoperative Management}

Generally, MIGS in combination with cataract extraction can be managed with the same postoperative regimen as phacoemulsification alone. For most surgeons, this includes intracameral and/or topical antibiotic therapy, a topical steroid taper, and possibly a topical nonsteroidal anti-inflammatory eye drop. There is no clear evidence that procedures to excise, ablate, open, or stent the trabecular meshwork do better with postoperative miotics [61], and it is not our practice to use them. Following ECP, we often lengthen the duration of the steroid taper to 2 or more months, tailored to the individual's anterior chamber inflammation.

There is no clear evidence to guide reduction in IOP lowering therapy following MIGS. We find it helpful to discuss with patients and document in the chart whether the goal of MIGS is to reduce IOP on their current eye drop regimen or maintain their target IOP on a reduced eye drop regimen. This discussion often informs eye drop management in the early postoperative period. We tend to continue the preoperative IOP lowering regimen for the first postoperative week to blunt IOP elevation from inflammation or steroid response and reduce the regimen starting at the one week follow-up visit.

\section{MIGS Failures}

MIGS surgery directed at the trabecular or suprachoroidal outflow pathways has not been shown to have deleterious effects on subsequent incisional glaucoma surgery. Whether the same is true of subconjunctival MIGS is an area of considerable debate. In a small series of 8 eyes, trabeculectomy following a failed XEN gel stent was technically feasible but authors were concerned that the single digit IOPs (6 patients) and hypotony complications ( 2 patients) were more common than expected following trabeculectomy. They hypothesized this might be related to the cumulative exposure to mitomycin c [62].

\section{Future Work}

The emergence of MIGS has dramatically altered the glaucoma treatment paradigm over the last decade, and more research is needed to help answer remaining questions. Welldesigned, large studies are needed to compare the efficacy of the various MIGS options and identify whether certain patient-specific or eye-specific features make a particular surgery more efficacious. Similarly, the role of combination MIGS should be investigated further.

To our knowledge, MIGS trials to date have not been designed to assess glaucoma progression and are simply based on the abundance of prior literature showing that reduction in IOP slows glaucomatous progression. Including structure and function metrics in future studies and databases will add value.

Finally, questionnaires to better understand quality of life following MIGS are in development and will lead to patient reported outcome measures for MIGS [63], which will likely be included in future study designs.

\section{Conclusions}

The MIGS space has experienced tremendous growth in recent years. On average, MIGS is low risk, modestly efficacious, and can be readily combined with cataract surgery to reduce IOP and/or drop burden in patients with concurrent glaucoma and cataract. Future research to guide MIGS selection and highlight its role in improving patient quality of life will further our ability to deliver excellent, personalized, and sight-preserving glaucoma care.

\section{Compliance with Ethical Standards}

Conflict of Interest The authors declare that they have no conflicts of interest.

Human and Animal Rights and Informed Consent This article does not contain any studies with human or animal subjects performed by any of the authors. 
Open Access This article is distributed under the terms of the Creative Commons Attribution 4.0 International License (http:// creativecommons.org/licenses/by/4.0/), which permits unrestricted use, distribution, and reproduction in any medium, provided you give appropriate credit to the original author(s) and the source, provide a link to the Creative Commons license, and indicate if changes were made.

\section{References}

Papers of particular interest, published recently, have been highlighted as:

- Of importance

•- Of major importance

1. Quigley HA, Broman AT. The number of people with glaucoma worldwide in 2010 and 2020. Br J Ophthalmol. 2006;90(3):262-7. https://doi.org/10.1136/bjo.2005.081224.

2. Tham Y-C, Li X, Wong TY, Quigley HA, Aung T, Cheng C-Y. Global prevalence of glaucoma and projections of glaucoma burden through 2040: a systematic review and meta-analysis. Ophthalmology. 2014;121(11):2081-90. https://doi.org/10.1016/j. ophtha.2014.05.013

3. Boland MV, Ervin A-M, Friedman DS, et al. Comparative effectiveness of treatments for open-angle glaucoma: a systematic review for the U.S. Preventive Services Task Force. Ann Intern Med. 2013;158(4):271-9. https://doi.org/10.7326/0003-4819-1584-201302190-00008.

4. Janz NK, Wren PA, Lichter PR, Musch DC, Gillespie BW, Guire KE. Quality of life in newly diagnosed glaucoma patients: the Collaborative Initial Glaucoma Treatment Study. Ophthalmology. 2001;108(5):887-97 discussion 898.

5. Varma R, Lee PP, Goldberg I, Kotak S. An assessment of the health and economic burdens of glaucoma. Am J Ophthalmol. 2011;152(4):515-22. https://doi.org/10.1016/j.ajo.2011.06.004.

6. Medeiros FA, Gracitelli CPB, Boer ER, Weinreb RN, Zangwill LM, Rosen PN. Longitudinal changes in quality of life and rates of progressive visual field loss in glaucoma patients. Ophthalmology. 2015;122(2):293-301. https://doi.org/10.1016/j. ophtha.2014.08.014.

7. Nordmann J-P, Auzanneau N, Ricard S, Berdeaux G. Vision related quality of life and topical glaucoma treatment side effects. Health Qual Life Outcomes. 2003;1:75. https://doi.org/10.1186/14777525-1-75.

8.• Saheb H, Ahmed IIK. Micro-invasive glaucoma surgery: current perspectives and future directions. Curr Opin Ophthalmol. 2012;23(2):96-104. https://doi.org/10.1097/ICU. 0b013e32834ffle 7 This article helped to provide a definition for MIGS.

9. Chang TC, Vanner EA, Parrish RK. Glaucoma surgery preferences when the surgeon adopts the role of the patient. Eye Lond Engl. 2019. https://doi.org/10.1038/s41433-019-0452-9 A survey of American glaucoma specialists demonstrating that about half of surgeons would prefer to have MIGS if their own eye in the setting of POAG and progressive visual field loss in need of surgery.

10. Lavia C, Dallorto L, Maule M, Ceccarelli M, Fea AM. Minimallyinvasive glaucoma surgeries (MIGS) for open angle glaucoma: a systematic review and meta-analysis. PloS One. 2017;12(8): e0183142. https://doi.org/10.1371/journal.pone.0183142 A large systematic review and meta-analysis on the safety and efficacy of available MIGS options.
11. iStent inject Trabecular Micro-Bypass System (Model G2-M-IS) P170043. https://www.fda.gov/medical-devices/recently-approveddevices/istent-inject-trabecular-micro-bypass-system-model-g2-mp170043. Published May 18, 2019.

12. Wang SY, Singh K, Stein JD, Chang RT. Ocular antihypertensive medication use after iStent implantation concurrent with cataract surgery vs cataract surgery alone in a large US health care claims database. JAMA Ophthalmol. September 2018. https://doi.org/10. 1001/jamaophthalmol.2018.4461.

13. Samuelson TW, Sarkisian SR, Lubeck DM, et al. Prospective, randomized, controlled pivotal trial of iStent inject trabecular microbypass in primary open-angle glaucoma and cataract: two-year results. Ophthalmology. March 2019. https://doi.org/10.1016/j. ophtha.2019.03.006.

14. Gillmann K, Bravetti GE, Mermoud A, Mansouri K. A prospective analysis of iStent inject microstent positioning: Schlemm's canal dilatation and intraocular pressure correlations. J Glaucoma. 2019. https://doi.org/10.1097/IJG.0000000000001273.

15. Le JT, Bicket AK, Wang L, Li T. Ab interno trabecular bypass surgery with iStent for open-angle glaucoma. Cochrane Database Syst Rev. 2019;3:CD012743. https://doi.org/10.1002/14651858. CD012743.pub2.

16. Huang AS, Penteado RC, Papoyan V, Voskanyan L, Weinreb RN. Aqueous angiographic outflow improvement after trabecular microbypass in glaucoma patients. Ophthalmol Glaucoma. 2019;2(1):11-21. https://doi.org/10.1016/j.ogla.2018.11.010.

17.• Samuelson TW, Chang DF, Marquis R, et al. A Schlemm Canal Microstent for Intraocular Pressure Reduction in Primary OpenAngle Glaucoma and Cataract: The HORIZON Study. Ophthalmology. 2019;126(1):29-37. https://doi.org/10.1016/j. ophtha.2018.05.012 A prospective, multicenter, randomized controlled trial and the largest MIGS trial to date demonstrating the two year results of the Hydrus microstent together with cataract extraction.

18. Yook E, Vinod K, Panarelli JF. Complications of micro-invasive glaucoma surgery. Curr Opin Ophthalmol. 2018;29(2):147-54. https://doi.org/10.1097/ICU.0000000000000457.

19. Posarelli C, Ortenzio P, Ferreras A, Toro MD, Passani A, Loiudice $\mathrm{P}$, et al. Twenty-four-hour contact lens sensor monitoring of aqueous humor dynamics in surgically or medically treated glaucoma patients. J Ophthalmol. 2019;2019:9890831. https://doi.org/10. 1155/2019/9890831.

20. Asrani S, Zeimer R, Wilensky J, Gieser D, Vitale S, Lindenmuth K. Large diurnal fluctuations in intraocular pressure are an independent risk factor in patients with glaucoma. J Glaucoma. 2000;9(2): 134-42.

21. Sieck EG, Epstein RS, Kennedy JB, et al. Outcomes of Kahook dual blade goniotomy with and without phacoemulsification cataract extraction. Ophthalmol Glaucoma. 2018;1(1):75-81. https:// doi.org/10.1016/j.ogla.2018.06.006.

22. Hirabayashi MT, King JT, Lee D, An JA. Outcome of phacoemulsification combined with excisional goniotomy using the Kahook dual blade in severe glaucoma patients at 6 months. Clin Ophthalmol Auck1 NZ. 2019;13:715-21. https://doi.org/10. 2147/OPTH.S196105.

23. Dorairaj S, Tam MD. Kahook dual blade excisional goniotomy and goniosynechialysis combined with phacoemulsification for angle closure glaucoma: 6-month results. J Glaucoma. 2019. https://doi. org/10.1097/IJG.0000000000001256.

24. Dorairaj SK, Kahook MY, Williamson BK, Seibold LK, ElMallah MK, Singh IP. A multicenter retrospective comparison of goniotomy versus trabecular bypass device implantation in glaucoma patients undergoing cataract extraction. Clin Ophthalmol Auckl NZ. 2018;12:791-7. https://doi.org/10.2147/OPTH.S158403.

25. Ahmed SF, Bhatt A, Schmutz M, Mosaed S. Trabectome outcomes across the spectrum of glaucoma disease severity. Graefes Arch 
Clin Exp Ophthalmol Albrecht Von Graefes Arch Klin Exp Ophthalmol. 2018;256(9):1703-10. https://doi.org/10.1007/ s00417-018-4023-8.

26. Esfandiari H, Shah P, Torkian P, Conner IP, Schuman JS, Hassanpour K, et al. Five-year clinical outcomes of combined phacoemulsification and trabectome surgery at a single glaucoma center. Graefes Arch Clin Exp Ophthalmol Albrecht Von Graefes Arch Klin Exp Ophthalmol. 2019;257(2):357-62. https://doi.org/ 10.1007/s00417-018-4146-y.

27. Nazarali SA, Damji KF. Ab interno trabeculectomy with Trabectome: outcomes in African American versus Caucasian patients. Can J Ophthalmol J Can Ophtalmol. 2018;53(4):361-4. https://doi.org/10.1016/j.jcjo.2017.10.018.

28. Grover DS, Godfrey DG, Smith O, Feuer WJ, Montes de Oca I, Fellman RL. Gonioscopy-assisted transluminal trabeculotomy, ab interno trabeculotomy: technique report and preliminary results. Ophthalmology. 2014;121(4):855-61. https://doi.org/10.1016/j. ophtha.2013.11.001.

29. Grover DS, Smith O, Fellman RL, Godfrey DG, Gupta A, Montes de Oca I, et al. Gonioscopy-assisted transluminal trabeculotomy: an ab interno circumferential trabeculotomy: 24 months follow-up. J Glaucoma. 2018;27(5):393-401. https://doi.org/10.1097/IJG. 0000000000000956.

30. Aktas Z, Ozmen MC, Atalay HT, Ucgul AY. Evaluation of episcleral venous fluid wave during gonioscopy assisted transluminal trabeculotomy in patients with advanced glaucoma. Eye Lond Engl. 2019;33(4):668-73. https://doi.org/10.1038/s41433-0180254-5.

31. Riva I, Brusini P, Oddone F, Michelessi M, Weinreb RN, Quaranta L. Canaloplasty in the treatment of open-angle glaucoma: a review of patient selection and outcomes. Adv Ther. 2019;36(1):31-43. https://doi.org/10.1007/s12325-018-0842-6.

32. Gallardo MJ, Supnet RA, Ahmed IIK. Viscodilation of Schlemm's canal for the reduction of IOP via an ab-interno approach. Clin Ophthalmol Auckl NZ. 2018;12:2149-55. https://doi.org/10.2147/ OPTH.S177597.

33. Use of the OMNI $®$ surgical system in combination with cataract extraction in open angle glaucoma. https://www.clinicaltrials.gov/ ct $2 /$ show/NCT03861169?term $=$ sight+sciences\&rank $=2$. Published March 4, 2019. Accessed June 8, 2019.

34. Green W, Lind JT, Sheybani A. Review of the Xen gel stent and InnFocus MicroShunt. Curr Opin Ophthalmol. 2018;29(2):162-70. https://doi.org/10.1097/ICU.0000000000000462.

35. De Gregorio A, Pedrotti E, Russo L, Morselli S. Minimally invasive combined glaucoma and cataract surgery: clinical results of the smallest ab interno gel stent. Int Ophthalmol. 2018;38(3):112934. https://doi.org/10.1007/s10792-017-0571-x.

36. Hohberger B, Welge-Lüßen U-C, Lämmer R. MIGS: therapeutic success of combined Xen gel stent implantation with cataract surgery. Graefes Arch Clin Exp Ophthalmol Albrecht Von Graefes Arch Klin Exp Ophthalmol. 2018;256(3):621-5. https://doi.org/ 10.1007/s00417-017-3895-3.

37. Ibáñez-Muñoz A, Soto-Biforcos VS, Rodríguez-Vicente L, et al. XEN implant in primary and secondary open-angle glaucoma: a 12-month retrospective study. Eur J Ophthalmol. 2019: 1120672119845226. https://doi.org/10.1177/1120672119845226.

38. Marques RE, Ferreira NP, Sousa DC, Pinto J, Barata A, Sens P, et al. Glaucoma gel implant learning curve in a teaching tertiary hospital. J Glaucoma. 2019;28(1):56-60. https://doi.org/10.1097/ IJG.0000000000001107.

39. Heidinger A, Schwab C, Lindner E, Riedl R, Mossböck G. A retrospective study of 199 Xen 45 stent implantations from 2014 to 2016. J Glaucoma. 2019;28(1):75-9. https://doi.org/10.1097/IJG. 0000000000001122 .

40. Ibáñez-Muñoz A, Soto-Biforcos VS, Chacón-González M, RúaGalisteo O, Arrieta-Los Santos A, Lizuain-Abadía ME, et al.
One-year follow-up of the XEN® implant with mitomycin-C in pseudoexfoliative glaucoma patients. Eur J Ophthalmol. 2019;29(3):309-14. https://doi.org/10.1177/1120672118795063.

41. Dar N, Sharon T, Hecht I, Kalev-Landoy M, Burgansky-Eliash Z. Efficacy and safety of the ab interno gelatin stent in severe pseudoexfoliation glaucoma compared to non-pseudoexfoliation glaucoma at 6 months. Eur J Ophthalmol. 2019: 1120672119848277. https://doi.org/10.1177/1120672119848277.

42. Reitsamer H, Sng C, Vera V, Lenzhofer M, Barton K, Stalmans I, et al. Two-year results of a multicenter study of the ab interno gelatin implant in medically uncontrolled primary open-angle glaucoma. Graefes Arch Clin Exp Ophthalmol Albrecht Von Graefes Arch Klin Exp Ophthalmol. 2019;257(5):983-96. https://doi.org/ 10.1007/s00417-019-04251-z.

43. Widder RA, Dietlein TS, Dinslage S, Kühnrich P, Rennings C, Rössler G. The XEN45 gel stent as a minimally invasive procedure in glaucoma surgery: success rates, risk profile, and rates of resurgery after 261 surgeries. Graefes Arch Clin Exp Ophthalmol Albrecht Von Graefes Arch Klin Exp Ophthalmol. 2018;256(4): 765-71. https://doi.org/10.1007/s00417-018-3899-7.

44. Lenzhofer M, Kersten-Gomez I, Sheybani A, et al. Four-year results of a minimally invasive transscleral glaucoma gel stent implantation in a prospective multi-centre study. Clin Exp Ophthalmol. 2018. https://doi.org/10.1111/ceo.13463.

45. Uram M. Ophthalmic laser microendoscope ciliary process ablation in the management of neovascular glaucoma. Ophthalmology. 1992;99(12):1823-8.

46. Edmiston AM, SooHoo JR, Seibold LK, Kahook MY, Palestine AG, Pantcheva MB. Postoperative inflammation after endoscopic cyclophotocoagulation: racial distribution and effect on outcomes. J Glaucoma. 2018;27(3):266-8. https://doi.org/10.1097/IJG. 0000000000000884 A novel study demonstrating the association between race and prolonged inflammation following ECP.

47. Lin MM, Rageh A, Turalba AV, Lee H, Falkenstein IA, Hoguet AS, et al. Differential efficacy of combined phacoemulsification and endocyclophotocoagulation in open-angle glaucoma versus angleclosure glaucoma. J Glaucoma. 2019;28(5):473-80. https://doi.org/ 10.1097/IJG.0000000000001225.

48. Tóth M, Shah A, Hu K, Bunce C, Gazzard G. Endoscopic cyclophotocoagulation (ECP) for open angle glaucoma and primary angle closure. Cochrane Database Syst Rev. 2019;2:CD012741. https://doi.org/10.1002/14651858.CD012741.pub2.

49. Chen MF, Kim CH, Coleman AL. Cyclodestructive procedures for refractory glaucoma. Cochrane Database Syst Rev. 2019;3: CD012223. https://doi.org/10.1002/14651858.CD012223.pub2.

50. Le C, Kazaryan S, Hubbell M, Zurakowski D, Ayyala RS. Surgical outcomes of phacoemulsification followed by iStent implantation versus goniotomy with the Kahook dual blade in patients with mild primary open-angle glaucoma with a minimum of 12-month follow-up. J Glaucoma. 2019;28(5):411-4. https://doi.org/10.1097/ IJG.0000000000001143.

51.• IIK A, Fea A, Au L, et al. A prospective randomized trial comparing Hydrus and iStent micro-invasive glaucoma glaucoma surgery implants for standalone treatment of open-angle glaucoma: the COMPARE study. Ophthalmology. 2019. https://doi.org/10.1016/ j.ophtha.2019.04.034 The first prospective, multicenter, randomized clinical trial comparing the efficacy of two. MIGS devices: the Hydrus microstent and two iStent inject stents.

52. Lee GA, Porter AJ, Vincent RA, Makk J, Vincent SJ. Combined phacoemulsification and microinvasive glaucoma surgery in comparison to phacoemulsification alone for open angle glaucoma. Eye Lond Engl. 2019:1-7. https://doi.org/10.1038/s41433-019-0459-2.

53. Esfandiari H, Taubenslag K, Shah P, Goyal S, Weiner AJ, Severson ML, et al. Two-year data comparison of ab interno trabeculectomy and trabecular bypass stenting using exact matching. J Cataract 
Refract Surg. 2019;45(5):608-14. https://doi.org/10.1016/j.jcrs. 2018.12.011.

54. Myers JS, Masood I, Hornbeak DM, Belda JI, Auffarth G, Jünemann A, et al. Prospective evaluation of two iStent ${ }^{\circledR}$ trabecular stents, one iStent supra ${ }^{\circledR}$ suprachoroidal stent, and postoperative prostaglandin in refractory glaucoma: 4-year outcomes. Adv Ther. 2018;35(3):395-407. https://doi.org/10.1007/s12325-018-0666-4.

55. Ferguson TJ, Swan R, Sudhagoni R, Berdahl JP. Microbypass stent implantation with cataract extraction and endocyclophotocoagulation versus microbypass stent with cataract extraction for glaucoma. J Cataract Refract Surg. 2017;43(3):377-82. https://doi.org/10.1016/j.jcrs.2016.12. 020 .

56. Multicenter investigation of the Glaukos ${ }^{\circledR}$ suprachoroidal stent model G3 in conjunction with cataract surgery. https:// clinicaltrials.gov/ct2/show/NCT01461278. Published March 8, 2019. Accessed June 8, 2019.

57. Broadway DC, Grierson I, O'Brien C, Hitchings RA. Adverse effects of topical antiglaucoma medication. II. The outcome of filtration surgery. Arch Ophthalmol Chic Ill 1960. 1994;112(11):144654.

58. Lavin MJ, Wormald RP, Migdal CS, Hitchings RA. The influence of prior therapy on the success of trabeculectomy. Arch Ophthalmol Chic Ill 1960. 1990;108(11):1543-8.

59. Tailor R, Batra R, Mohamed S. A national survey of glaucoma specialists on the preoperative (trabeculectomy) management of the ocular surface (.). Semin Ophthalmol. 2016;31(6):519-25. https://doi.org/10.3109/08820538.2014.986585.

60. Gazzard G, Konstantakopoulou E, Garway-Heath D, Garg A, Vickerstaff V, Hunter R, et al. Selective laser trabeculoplasty versus eye drops for first-line treatment of ocular hypertension and glaucoma (LiGHT): a multicentre randomised controlled trial. Lancet Lond Engl. 2019;393(10180):1505-16. https://doi.org/10.1016/ S0140-6736(18)32213-X.

61. Esfandiari H, Hassanpour K, Yaseri M, Loewen NA. Extended pharmacological miosis is superfluous after glaucoma angle surgery: a retrospective study. F1000Research. 2018;7:178. https:// doi.org/10.12688/f1000research.13756.1.

62. Gizzi C, Mohamed-Noriega J, Elkarmouty A, Scott A. Trabeculectomy following failed ab interno gelatin microstent: case series. J Glaucoma. 2018;27(10):e168-73. https://doi.org/10. 1097/IJG.0000000000001000.

63. Patient reported outcomes for minimally invasive glaucoma surgery (MIGS). https://www.fda.gov/science-research/advancingregulatory-science/patient-reported-outcomes-minimally-invasiveglaucoma-surgery-migs. Published March 29, 2018. Accessed May 11, 2019.

Publisher's Note Springer Nature remains neutral with regard to jurisdictional claims in published maps and institutional affiliations. 